\title{
Lie symmetry analysis of the Hanta-epidemic systems
}

\author{
Mevlude Yakit Ongun a,*, Mehmet Kocabiyik ${ }^{\mathrm{b}}$ \\ a Suleyman Demirel University, Department of Mathematics, Isparta, Turkey. \\ b Suleyman Demirel University, Graduate School of Natural and Applied Sciences, Isparta, Turkey.
}

\begin{abstract}
We consider a model for the fatal Hanta-virus infection among mice. Lie symmetry analysis is applied to find general solutions to Hanta-virus model, which is also known as Abramson-Kenkre model. Besides the solution for the version with derivatives of fractional order, we investigate the model also by using the Lie symmetry method. The basic point of view for both situations will be logistic differential equation, created for total population. (c)2017 All rights reserved.
\end{abstract}

Keywords: Lie symmetries, logistic differential equation, Hanta epidemics, fractional order differential equation. 2010 MSC: 34A05, 34C14, 92D30.

\section{Introduction}

Hanta-virus causes a fatal contagious disease spreading among mice and the reason for being fatal is the fact that the viruses genetically contain one chain RNA. This virus has various types, and has been first discovered in South Korea in Hanta river. In this article, Abramson-Kenkre mathematical model [2] will be used to examine, understand and analyze Hanta-virus dynamics better. This model takes into account the spatial and temporal characteristics of Hanta-virus infection. Allen et al. [4] suggested an ordinarydifferential-equation model for virus infection. Allen et al. [5] developed two new models for Hanta-virus infections among male and female rodents. Abramson-Kenkre model is a system of nonlinear differential equations that defines virus transition from infected mice to susceptible ones. This model, defined by partial differential equation in Abramson et al. [3], separates the whole mice population in two groups: susceptible and infected.

There is a version of Hanta-virus infection model with ordinary derivatives [2, 4], in the form

$$
\begin{aligned}
\frac{d M_{s}}{d t} & =b\left(M_{s}+M_{i}\right)-c M_{s}-\frac{M_{s}\left(M_{s}+M_{i}\right)}{K}-a M_{s} M_{i}, \\
\frac{d M_{i}}{d t} & =-c M_{i}-\frac{M_{i}\left(M_{s}+M_{i}\right)}{K}+a M_{s} M_{i},
\end{aligned}
$$

where

\footnotetext{
*Corresponding author

Email addresses: mevludeyakit@sdu.edu.tr (Mevlude Yakit Ongun), dogangunesmehmet@hotmail.com (Mehmet Kocabiyik)
} doi:10.22436/jmcs.017.02.16 
$M_{s}:$ Mice population without disease;

$M_{i}$ : Mice population with disease;

a: infection rate;

b: birth rate;

c: death rate;

K: Environment contagion capacity.

In this model, the infection process is ignored, and the whole population is split into two groups: sensitive mice and mice with disease.

In system (1.1) and (1.2), if $M=M_{s}+M_{i}$ is the total population, then

$$
\frac{d M}{d t}=(b-c) M-\frac{M^{2}}{K},
$$

so we obtain the logistic differential equation. In the next phases of this article, with the use of general solution of logistic differential equation with Lie symmetry method, we will calculate $M_{s}$ and $M_{i}$ populations. Besides, the version of the Hanta-virus model with derivatives of fractional order is

$$
\begin{aligned}
& D_{t}^{\alpha} M_{s}=b\left(M_{s}+M_{i}\right)-c M_{s}-\frac{M_{s}\left(M_{s}+M_{i}\right)}{K}-a M_{s} M_{i}, \\
& D_{t}^{\alpha} M_{i}=-c M_{i}-\frac{M_{i}\left(M_{s}+M_{i}\right)}{K}+a M_{s} M_{i},
\end{aligned}
$$

where $D_{t}^{\alpha}$ denotes the fractional derivative operator with respect to the origin, according to Caputo's definition [29]. $M_{s}(t)$ and $M_{i}(t)$ are the activator and inhibitor variables, respectively. Fractional derivatives are used to describe non-homogeneous character of ecosystems, with respect to presence of competitors. The parameter $\alpha$ denotes the density of competitor species in the systems [1]. When $0<\alpha<1$, competitor population varies, and for $\alpha>1$, it increases.

These are some suggestions about the solution of Hanta epidemic model $[1,3,9,10,15,18-20,24,30$, 33, 34].

This paper is organized as follows: in Section 2, we define some basic definitions and theorems related with Lie symmetry transformation. Section 3 includes basic concepts and prolongation formula for fractional-order differential equations. In Section 4, we find general solutions of the Hanta-virus model, both of ordinary and fractional orders. The results are illustrated by some graphics and figures.

\section{Basic definitions and concepts}

For this section, these are the important books and papers about Lie groups and Lie symmetry transformation: $[7,8,11-14,16,21-23,27,28,32]$.

\subsection{Lie groups with one parameter}

Let

$$
\phi: \mathbb{R}^{2} \times a \rightarrow \mathbb{R}, \quad \psi: \mathbb{R}^{2} \times a \rightarrow \mathbb{R},
$$

where for all $a \in \mathbb{R}$, the parameters $\phi$ and $\psi$ are analytical functions

$$
\phi(x, y, a)=x_{1}, \quad \text { and } \quad \psi(x, y, a)=y_{1},
$$

and using $\mathrm{T}_{\mathrm{a}}: \mathbb{R}^{2} \rightarrow \mathbb{R}^{2}$

$$
(x, y) \rightarrow T_{a}(x, y)=(\phi(x, y, a), \psi(x, y, b))=\left(x_{1}, y_{1}\right)
$$

the set

$$
\mathrm{G}=\left[\mathrm{T}_{\mathrm{a}} \mid \quad \mathrm{a} \in \mathbb{R}\right],
$$

will be defined. If the set (2.2) satisfies the group axioms, then it is named as one-parameter Lie group. The functions $\phi$ and $\psi$ in the definition of Lie are named as global form of group (finite form). If we take 
an arbitrary point $(x, y)$ and expand (2.1) in the Taylor series about $a=0$, we obtain

$$
\begin{aligned}
& x_{1}=x+a \xi(x, y)+0\left(a^{2}\right), \\
& y_{1}=y+a \eta(x, y)+0\left(a^{2}\right),
\end{aligned}
$$

which is named infinitesimal transformations of the Lie group transformations, and $\xi$ and $\eta$ are infinitesimals of the group and are defined by [27]

$$
\begin{aligned}
\xi(x, y) & =\left(\frac{\partial x_{1}}{\partial a}\right)_{a=0}, \\
\eta(x, y) & =\left(\frac{\partial y_{1}}{\partial a}\right)_{a}=0 .
\end{aligned}
$$

We can use differential operator

$$
L=\xi(x, y) \frac{\partial}{\partial x}+\eta(x, y) \frac{\partial}{\partial y},
$$

to observe a smooth function change under the influence of an infinitesimal. The operator $L$ is called an infinitesimal generator of Lie group or the Lie operator.

\subsection{Canonical form and variables}

If $\xi(x, y)=0$ and $\eta(x, y)=0$, then $(x, y)$ will be a fixed point under the influence of infinitesimal transformation, so it will be fixed under the influence of all variables of the group. These points are called absolute invariant points.

Theoretically, it is always possible to find a variable transformation which can transform a parametrized Lie group operator in any wanted structure. Especially, to reduce the Lie operator the translation along $y$ axis which means that the equations that will be integrated; when the operator has the form $\mathrm{L}=\frac{\partial}{\partial y}$ then

$$
\begin{aligned}
& \xi \frac{\partial \mathbf{x}}{\partial x}+\frac{\partial \mathbf{x}}{\partial y}=0 \\
& \xi \frac{\partial \mathbf{y}}{\partial x}+\frac{\partial \mathbf{y}}{\partial y}=1
\end{aligned}
$$

The Lie operator in the form $\mathbf{L}=\frac{\partial}{\partial y}$ is called the operator in canonical form, and the variables which reduce it to this form are called canonical variables. Every Lie operator $\mathbf{L}=\frac{\partial}{\partial y}$ can be reduced to the canonical form

$$
\frac{d x}{\xi}=\frac{d y}{\eta}
$$

To find the canonical variables, we should solve first-order differential equation (2.5) and we should solve equations (2.3) and (2.4), [1-16, 20-23, 26-29, 33].

2.3. Solution of the ordinary differential equations of the first degree by using transformation of Lie symmetry

To set the conditions of symmetry for ordinary differential equations of the first order, we consider

$$
y^{\prime}=f(x, y)
$$

As known, symmetry transformations leave new coordinates of differential equations without changing them. So,

$$
\mathrm{T}_{\epsilon}:(x, y) \rightarrow\left(x_{1}, y_{1}\right)=\left(x_{1}(x, y, \epsilon), \quad y_{1}(x, y, \epsilon)\right), \quad \in \in \mathbb{R} .
$$

Transformation (2.7) is symmetry for the equation; in that case it is called symmetry condition for the 
equation given by

$$
\frac{d y_{1}}{d x_{1}}=f\left(x_{1}, y_{1}\right)
$$

To make this condition more useful, $\mathrm{D}_{x}$ is taken to show total derivative in $x$ direction,

$$
D_{x}=\partial_{x}+y^{\prime} \partial_{y}+y^{\prime \prime} \partial_{y^{\prime}}+\cdots .
$$

So, for equation (2.6) symmetry condition will be (2.8)

$$
\frac{d y_{1}}{d x_{1}}=\frac{D_{x} y_{1}}{D_{x} x_{1}}=\frac{y_{1 x}+y^{\prime} y_{1 y}}{x_{1 x}+y^{\prime} x_{1 y}}
$$

and

$$
\frac{y_{1 x}+y^{\prime} y_{1 y}}{x_{1 x}+y^{\prime} x_{1 y}}=f\left(x_{1}, y_{1}\right)
$$

Now let us consider an orbit for an $(x, y)$ point which is non-invariant. If we write, together for an orbit on $\left(x_{1}, y_{1}\right)$, the tangent vector under the Lie groups influence,

$$
\frac{d x_{1}}{d \epsilon}=\xi\left(x_{1}, y_{1}\right), \quad \frac{d y_{1}}{d \epsilon}=\eta\left(x_{1}, y_{1}\right),
$$

and for $x_{1}$ and $y_{1}$ Taylor series expansion with the given symmetry condition under the influence $y^{\prime}=$ $f(x, y)$, we will have the following ordinary differential equations linearized symmetry condition

$$
\eta_{x}+\left(\eta_{y}-\xi_{x}\right) f-\xi_{y} f^{2}=\xi f_{x}+\eta f_{y} .
$$

\subsection{Canonical coordinates for ordinary differential equations of the first degree.}

Let assume that we can find non-trivial symmetries for ordinary differential equation (2.6); these symmetries include translational Lie group in y-direction. Our main aim here is to pass to new coordinates with the help of symmetry transformations of differential equation (2.6). These new coordinates will lead us to a new differential equation which includes translations directed to the dependent variable. These new coordinates are called canonical coordinates. In this direction, we have

$$
(r, s)=(r(x, y), s(x, y)), \quad r_{x} s_{y}-r_{y} s_{x} \neq 0,
$$

If we take new coordinates which are given in (2.10), for the considered new coordinates, with the use of ordinary differential equation and chain rule on tangent vector on $(r, s)$ point, the result will be

$$
\xi(x, y) r_{x}+\eta(x, y) r_{y}=0, \quad \xi(x, y) s_{x}+\eta(x, y) s_{y}=1,
$$

where $(r, s)$ satisfies

(i) if $\xi \neq 0$ an integral of differential equation

$$
\frac{d y}{d x}=\frac{\eta(x, y)}{\xi(x, y)}
$$

can be found easily. The first integral of the above ordinary differential equation will be

$$
\phi(x, y)=c, \quad \phi_{y} \neq 0,
$$

and

$$
r=\phi(x, y), \quad s=\left.\left(\int \frac{d x}{\xi(x, y(x, r))}\right)\right|_{r=r(x, y)} .
$$


(ii) If $\xi=0$ (if these symmetries are not trivial ones, then $\eta=0$ ). From the first equation of (2.11), we can see that $r_{y}=0$. Simply we can find the canonical coordinates:

$$
r=x, \quad \text { and } s=\left.\left(\int \frac{d y}{\eta(r, y)}\right)\right|_{r=x} .
$$

So, by the help of canonical coordinates, the general solution of the ordinary differential equation (2.6) will be $\left(\frac{\mathrm{d} s}{\mathrm{dr}}=\Omega(r, s)\right)$

$$
s(x, y)-\int^{r(x, y)} \Omega(r) d r+c=0
$$

\section{Fractional Lie symmetry}

\subsection{Basic concept and definitions for fractional-order differential equations}

Some books and studies that we can use as the basis for fractional-order differential equations are $[6,17,18,25,26,29,31]$. Let us consider the differential equation with fractional derivative of order $\alpha$.

$$
D_{x}^{\alpha} y(x)=f(x, y)
$$

First, we will consider the Riemann-Liouville fractional derivative operator, that can be defined by

$$
D_{x}^{\alpha} y(x)=\frac{1}{\Gamma(1-\alpha)} \frac{d}{d x} \int_{0}^{x} \frac{y(t)}{(x-t)^{\alpha}} d t
$$

where $\Gamma($.$) is the Gamma function.$

The Caputo fractional derivative operator is

$$
D_{x}^{\alpha} y(x)=\frac{1}{\Gamma(1-\alpha)} \int_{0}^{x} \frac{y^{\prime}(t)}{(x-t)^{\alpha}} d t
$$

In real-world problems, Caputo derivative is more common since it produces better results with initial and boundary conditions. Here we will use the Leibniz rule for general fractional order [18]

$$
D_{x}^{\alpha}(f(x) g(x))=\sum_{n=0}^{\infty}\left(\left(\begin{array}{l}
\alpha \\
n
\end{array}\right)\right) D_{x}^{\alpha-n} f(x) g^{n}(x), \quad \alpha>0 .
$$

In accordance with Lie group theory, we have the following infinitesimal transformation expandings

$$
\begin{aligned}
x_{1} & =x+a \xi(x, y)+0\left(a^{2}\right), \\
y_{1} & =y+a \eta(x, y)+0\left(a^{2}\right), \\
y_{1}^{n} & =y^{n}(x)+a \eta^{n}(x, y)+0\left(a^{2}\right), \\
D_{x}^{\alpha} y_{1} & =D_{x}^{\alpha} y+a \eta^{n}(x, y)+0\left(a^{2}\right) .
\end{aligned}
$$

With the use of above equations, equation (3.2) will be infinitesimal generator which expanded in order $\mathrm{n}$,

$$
\eta^{n}=D\left(\eta^{(n-1)}\right)-y^{(n)} D(\xi)
$$

where $\mathrm{D}$ is total derivative operator as defined in the theory of ordinary differential equations. The formula (3.2) is called prolongation formula. 
Having in mind the definition of total derivative $D$ and $y^{n}=\frac{d^{n} y}{d x^{n}},(3.2)$ can be rewritten in the form

$$
\eta^{n}=D^{n}\left(\eta-\xi y^{(1)}\right)+\xi y^{(n+1)} .
$$

By using (3.3), we can write the prolongation formula for fractional derivatives of order $\alpha$ :

$$
\eta^{(n)}=D_{x}^{(\alpha)}\left(\eta-\xi y^{(1)}\right)+\xi D_{x}^{(\alpha+1)} y .
$$

Prolongation formula can be easily generalized for fractional equations of order $\alpha[17,18,21,25,28]$,

$$
\eta^{\alpha}=D_{x}^{\alpha} \eta+D_{x}^{\alpha}\left(D_{x}(\xi) y\right)+\xi D_{x}^{\alpha+1} y-D_{x}^{\alpha+1}(\xi y) .
$$

Considering the infinitesimal generators $x_{1}$ and $y_{1}$ given at the beginning, and symmetry group transformation of differential equation of fractional order, the corresponding symmetry condition can be written

$$
\left.\left(\eta^{\alpha}-\frac{\partial f}{\partial x} \xi-\frac{\partial f}{\partial y} \eta\right)\right|_{D_{x}^{\alpha} y=f(x, y)}=0,
$$

where $\eta^{\alpha}$ is from the prolongation formula, and, using the general Leibniz rule [18] it can be also written as

$$
\eta^{\alpha}=D_{x}^{\alpha} \eta+\sum_{n=0}^{\infty}\left(\begin{array}{l}
\alpha \\
n
\end{array}\right) \frac{n-\alpha}{n+1} D_{x}^{\alpha-n} y D_{x}^{n+1} \xi
$$

\section{Hanta-virus epidemics with Lie symmetry method}

\subsection{Hanta-virus epidemics with ordinary order}

Summing, side by side, equations (1.1) and (1.2) and using $M=M_{s}+M_{i}$, we get

$$
\frac{d M}{d t}=(b-c) M-\frac{M^{2}}{K}
$$

which is the logistic differential equation. From (1.3) and linear symmetry condition, we get tangent vector

$$
(\xi, \eta)=(1,0) .
$$

Since $\xi \neq 0$ and using canonical coordinates, we find

$$
\mathrm{r}=\mathrm{c}, \text { and } \mathrm{s}=\mathrm{x},
$$

and then, using the solution of corresponding ordinary differential equations, we find the solution

$$
M=\frac{K(b-c) e^{\left(C_{1} b-C_{1} c+b t-c t\right)}}{-1+e^{\left(C_{1} b-C_{1} c+b t-c t\right)}}
$$

where $C_{1}$ is an arbitrary constant.

Using (1.3), the fact $M-M_{i}=M_{s}$, and (4.1), we can rewrite (1.2) in the form of the following ordinary differential equation for $M_{i}$,

$$
\begin{aligned}
\frac{d M_{i}}{d t}= & -c M_{i}-\frac{M_{i}\left(\left(\frac{K(b-c) e^{\left(C_{1} b-C_{1} c+b t-c t\right)}}{-1+e^{\left(C_{1} b-C 1 c+b t-c t\right)}}-M_{i}\right)+M_{i}\right)}{K} \\
& +a\left(\frac{K(b-c) e^{\left(C_{1} b-C_{1} c+b t-c t\right)}}{-1+e^{\left(C_{1} b-C_{1} c+b t-c t\right)}}-M_{i}\right) M_{i} .
\end{aligned}
$$

From (4.2) and getting into account linear symmetry condition, with the help of the MAPLE 18 and GEM 
package program, we we can get the tangent vectors as

$$
\left.(\xi, \eta)=\left(0,-\left(e^{(t c)}-e^{\left(C_{1} b-C 1 c+b t\right)}\right)^{(-a K)} M_{i}^{2}\left(e^{(t c(a K+1))}\right)-e^{\left(a c K t\left(C_{1} b-C_{1} c+b t\right)\right.}\right)\right) .
$$

So the solution for $M_{i}$ will be

$$
M_{i}=\frac{\left(\frac{A}{e^{C_{1} c}}\right)^{a K} e^{C_{1} c}}{e^{a c K t} A\left(I+C_{2}\right)},
$$

where $C_{1}$ and $C_{2}$ are arbitrary constants,

$$
A=e^{C_{1} b} e^{t b}-e^{C_{1} c} e^{t c},
$$

and

$$
I=\int \frac{a e^{C_{1} c}\left(-A e^{-C_{1} c}\right)^{a K}}{e^{a c K t}} d t .
$$

We will find the solution for $M_{s}$ with the help of (4.3) and (4.1) like this

$$
M_{s}=\frac{K(b-c) e^{\left(C_{1} b-C_{1} c+b t-c t\right)}}{-1+e^{\left(C_{1} b-C_{1} c+b t-c t\right)}}-\frac{\left(\frac{A}{e^{C_{1} c}}\right)^{a K} e^{C_{1} c}}{e^{a c K t} A\left(I+C_{2}\right)} .
$$

So, we proved the following proposition.

Proposition 4.1. Let $\mathrm{b}>\mathrm{c}$ for $\mathrm{k} \leqslant \frac{\mathrm{b}}{\mathrm{a}(\mathrm{b}-\mathrm{c})}$ and $\mathrm{k} \geqslant \frac{\mathrm{b}}{\mathrm{a}(\mathrm{b}-\mathrm{c})}$,

(i) The Lie symmetry solution for the total population $M=M_{s}+M_{i}$, of equation (1.3) is given by

$$
M=\frac{K(b-c) e^{\left(C_{1} b-C_{1} c+b t-c t\right)}}{-1+e^{\left(C_{1} b-C_{1} c+b t-c t\right)}} ;
$$

(ii) The solutions for mice populations: $M_{s}$ without disease and $M_{i}$ with disease, are

$$
M_{s}=\frac{K(b-c) e^{\left(C_{1} b-C_{1} c+b t-c t\right)}}{-1+e^{\left(C_{1} b-C_{1} c+b t-c t\right)}}-\frac{\left(\frac{A}{e^{C_{1} c}}\right)^{a K} e^{C_{1} c}}{e^{a c K t} A\left(I+C_{2}\right)},
$$

and

$$
M_{i}=\frac{\left(\frac{A}{e^{C_{1} c}}\right)^{a K_{e} e^{C_{1} c}}}{e^{a \mathrm{akt}^{\prime} A\left(I+C_{2}\right)}}
$$

respectively, where

$$
A=e^{C_{1} b} e^{t b}-e^{C_{1} c} e^{t c}
$$

and

$$
\mathrm{I}=\int \frac{\mathrm{ae} \mathrm{C}_{1 \mathrm{c}}\left(-\mathrm{A} e^{-\mathrm{C}_{1} \mathrm{c}}\right)^{\mathrm{aK}}}{e^{\mathrm{acKt}}} \mathrm{dt}
$$

4.2. Hanta-virus epidemics model with fractional order

By a similar procedure as above, from equations (1.4) and (1.5) with derivatives of fractional order, we can get

$$
D_{t}^{\alpha} M=(b-c) M-\frac{M^{2}}{K}
$$

which is again the logistic differential equation, where $M=M_{i}+M_{s}$. The corresponding symmetry 
condition is

$$
\eta^{\alpha}-\eta\left((b-c)-\frac{2}{K} M\right)=0 .
$$

If we choose $\xi=\xi(t)$ and $\eta=p(t) M+q(t)$ and using the Leibniz rule for $\eta^{\alpha}$, after some necessarily complex procedure, we will get the following D1-D2-D3-D4 determination equations

$$
\begin{aligned}
& \text { D1 : } D_{t}^{\alpha} q(t)=(b-c) q(t), \\
& \text { D2 }: \frac{2}{\alpha(b-c) K} q(t)=\xi^{\prime}(t), \\
& \text { D3 }:-\alpha \xi^{\prime}(t)=p(t), \\
& \text { D4 }: p^{n}(t)+\frac{n-\alpha}{n+1} \xi^{(n+1)}(t)=0,
\end{aligned}
$$

where $n \in \mathbb{N}$. If D1 equation is solved by using [25], we will have

$$
q(t)=t^{\alpha-1} E_{\alpha, \alpha}\left((b-c) t^{\alpha}\right) C_{3},
$$

where $C_{3}$ is an integral constant and $E_{\alpha, \alpha}($.$) is a two parameter Mittag-Leffler function defined by$

$$
E_{\alpha, \beta}\left(t^{\alpha}\right)=\sum_{k=0}^{\infty} \frac{t^{\alpha k}}{\Gamma(\alpha k+\beta)} .
$$

The Mittag-Leffler function is an extension of the exponential function. When $\alpha=1$, the expression for $q(t)$ reduces to $(b-c) e^{t}$.

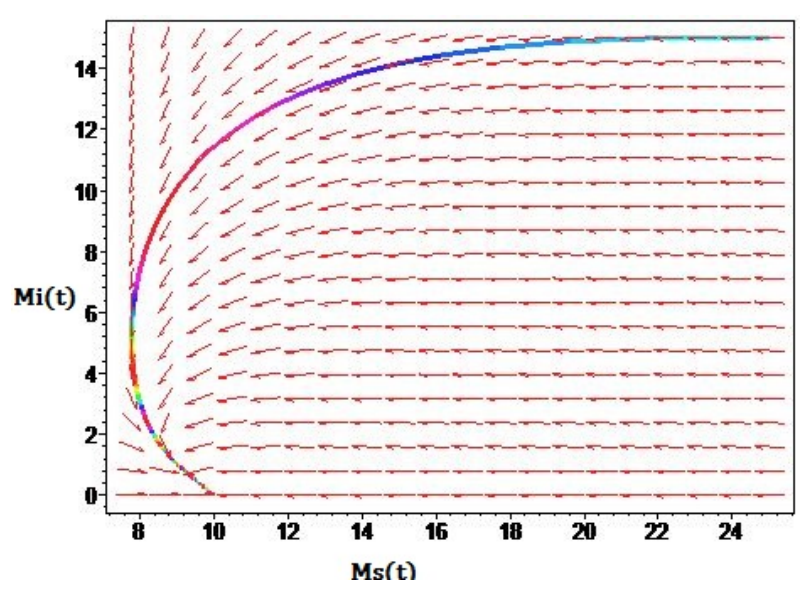

Figure 1: Phase portrait of equations (1.1) and (1.2).

Solution of D2, with the integral of $q(t)$ in $D 1$, is

$$
\xi(t)=C_{3} \frac{2}{K \alpha(b-c)} t^{\alpha} E_{\alpha, \alpha+1}\left((b-c) t^{\alpha}\right)+C_{4}
$$

we will find $\mathrm{C}_{4}$ as arbitrary integral constant. If we put $\xi^{\prime}(\mathrm{t})$ into D3, we get

$$
p(t)=C_{3} \frac{-2}{K(b-c)} t^{\alpha-1} E_{\alpha, \alpha}\left((b-c) t^{\alpha}\right),
$$

wherefrom we can easily find it. 


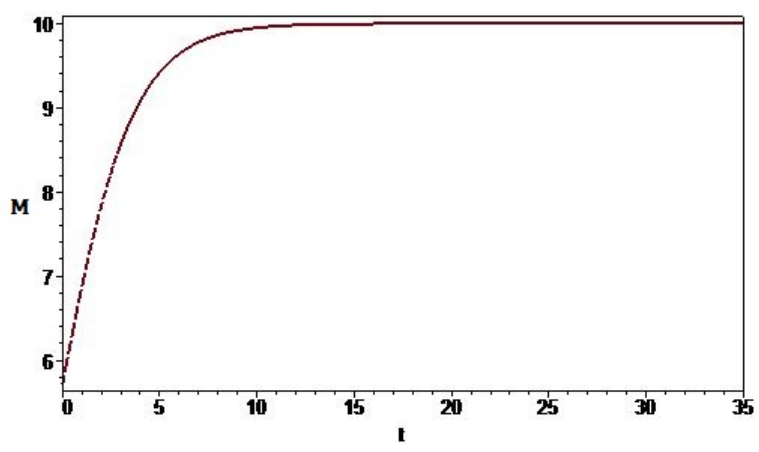

Figure 2: Solution of total population which is given by Equation (1.3).

The equation D4 is obviously ensured by the solution found. So the infinitesimal generator L will be

$$
\begin{aligned}
L= & {\left[C_{3} \frac{2}{K \alpha(b-c)} t^{\alpha} E_{\alpha, \alpha+1}\left((b-c) t^{\alpha}\right)+C_{4}\right] \frac{\partial}{\partial t} } \\
& +\left[C_{3} \frac{-2}{K(b-c)} t^{\alpha-1} E_{\alpha, \alpha}\left((b-c) t^{\alpha}\right) M+C_{3} t^{\alpha-1} E_{\alpha, \alpha}\left((b-c) t^{\alpha}\right)\right] \frac{\partial}{\partial M} .
\end{aligned}
$$

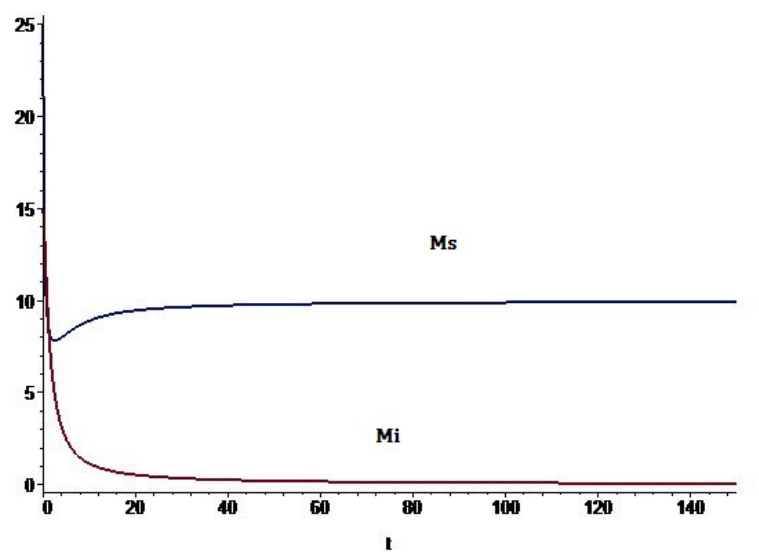

Figure 3: Solution of $M_{s}$ and $M_{i}$ which are given by equations (1.1) and (1.2).

If we put here $C_{3}=1$ and $C_{4}=0$ in infinitesimal generator, with the help of this differential operator and the equation given in (2.5), general solution will be,

$$
\begin{gathered}
\frac{d t}{\frac{2}{K \alpha(b-c)} t^{\alpha} E_{\alpha, \alpha+1}\left((b-c) t^{\alpha}\right)}, \\
\frac{d M}{\frac{-2}{K(b-c)} t^{\alpha-1} E_{\alpha, \alpha}\left((b-c) t^{\alpha}\right) M+t^{\alpha-1} E_{\alpha, \alpha}\left((b-c) t^{\alpha}\right)} .
\end{gathered}
$$

If we equate the above statements and then integrating the equation, the solution of $M$ will be obtained as follows:

$$
M=\frac{\left[\left(t^{\alpha} E_{\alpha, \alpha+1}\left((b-c) t^{\alpha}\right)^{-\alpha}\right)-1\right]}{-2 /(K(b-c))}+C_{5}
$$


where $C_{5}$ is an arbitrary constant. Using this solution and taking $M-M_{i}=M_{s}$, the new version of equation (1.5) will be

$$
\begin{aligned}
D_{t}^{\alpha} M_{i}= & -c M_{i}-\frac{M_{i}\left(\frac{\left[\left(t^{\alpha} E_{\alpha, \alpha+1}\left((b-c) t^{\alpha}\right)^{-\alpha}\right)-1\right]}{-2 /(K(b-c))}+C_{5}\right)}{K} \\
& +a\left(\frac{\left[\left(t^{\alpha} E_{\alpha, \alpha+1}\left((b-c) t^{\alpha}\right)^{-\alpha}\right)-1\right]}{-2 /(K(b-c))}+C_{5}-M_{i}\right) M_{i} .
\end{aligned}
$$

For this equation, if we apply symmetry condition and solve the determination equation, the tangent vector will be found as

$$
(\xi, \eta)=\left(\left(c+\frac{M}{K}-a M\right)^{\frac{-1}{\alpha}},\left(\left(c+\frac{M}{K}-a M\right)^{\frac{-1}{\alpha}-1}\left(M^{\prime}-a M^{\prime}\right) M_{i}\right),\right.
$$

where $M$ is the solution of fractional-order differential logistic equation with $M$ given in (4.6). Here, with the conclusion of integral procedure on both sides on the equation (2.5), the solution for $M_{i}$ will be found as

$$
\begin{aligned}
& M_{i}=\left(c+\frac{\frac{\left[\left(t^{\alpha} E_{\alpha, \alpha+1}\left((b-c) t^{\alpha}\right)^{-\alpha}\right)-1\right]}{-2 /(K(b-c))}+C_{5}}{K}\right. \\
&\left.-a\left(\frac{\left[\left(t^{\alpha} E_{\alpha, \alpha+1}\left((b-c) t^{\alpha}\right)^{-\alpha}\right)-1\right]}{-2 /(K(b-c))}+C_{5}\right)\right)+C_{6},
\end{aligned}
$$

where $\mathrm{C}_{6}$ is an arbitrary constant.
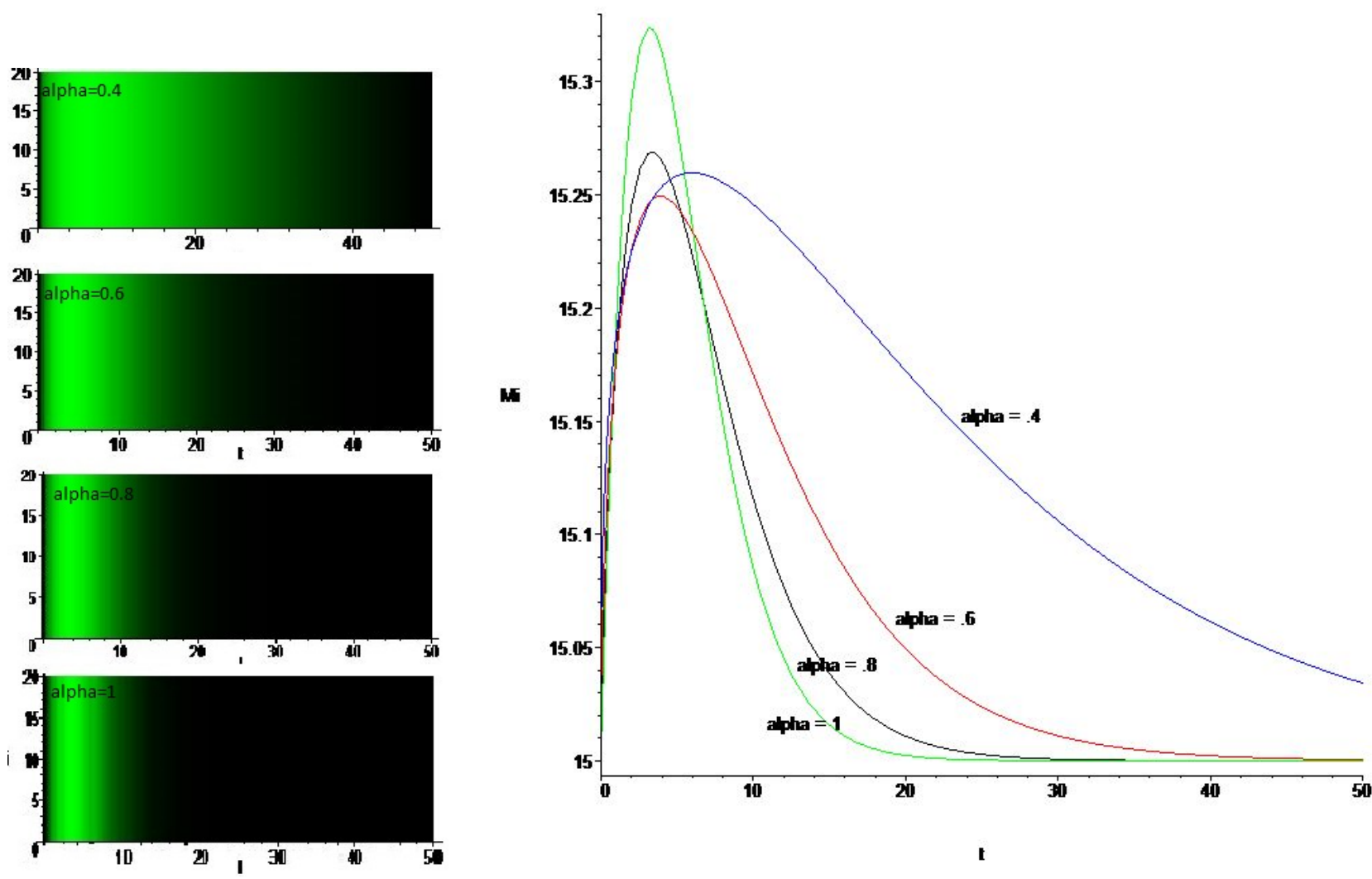

Figure 4: Density plots (left) and solutions of $M_{i}$ for different $\alpha$ values (right). 
With the help solution of (4.8) and using the logistic differential equation (4.6), we can get

$$
\begin{aligned}
M_{s}= & \frac{\left[\left(t^{\alpha} E_{\alpha, \alpha+1}\left((b-c) t^{\alpha}\right)^{-\alpha}\right)-1\right]}{-2 /(K(b-c))}+C_{5}-\left(c+\frac{\frac{\left[\left(t^{\alpha} E_{\alpha, \alpha+1}\left((b-c) t^{\alpha}\right)^{-\alpha}\right)-1\right]}{-2 /(K(b-c))}+C_{5}}{K}\right. \\
& \left.-a\left(\frac{\left[\left(t^{\alpha} E_{\alpha, \alpha+1}\left((b-c) t^{\alpha}\right)^{-\alpha}\right)-1\right]}{-2 /(K(b-c))}+C_{5}\right)\right)+C_{6} .
\end{aligned}
$$

So, we proved the following proposition.

\section{Proposition 4.2.}

(i) For the Lie symmetry solution for the total population in the version with derivatives of fractional order and under the conditions D1, D2, D3 and D4, we have

$$
M=\frac{\left[\left(t^{\alpha} E_{\alpha, \alpha+1}\left((b-c) t^{\alpha}\right)^{-\alpha}\right)-1\right]}{-2 /(K(b-c))}+C_{5}
$$

(ii) By using (i), and the fact $M=M_{s}+M_{i}$ we can find $M_{s}$ and $M_{i}$ :

$$
\begin{aligned}
M_{s}= & \frac{\left[\left(t^{\alpha} E_{\alpha, \alpha+1}\left((b-c) t^{\alpha}\right)^{-\alpha}\right)-1\right]}{-2 /(K(b-c))}+C_{5}-\left(c+\frac{\frac{\left[\left(t^{\alpha} E_{\alpha, \alpha+1}\left((b-c) t^{\alpha}\right)^{-\alpha}\right)-1\right]}{-2 /(K(b-c))}+C_{5}}{K}\right. \\
& \left.-a\left(\frac{\left[\left(t^{\alpha} E_{\alpha, \alpha+1}\left((b-c) t^{\alpha}\right)^{-\alpha}\right)-1\right]}{-2 /(K(b-c))}+C_{5}\right)\right)+C_{6},
\end{aligned}
$$

and

$$
\begin{aligned}
M_{i}= & \left(c+\frac{\frac{\left[\left(t^{\alpha} E_{\alpha, \alpha+1}\left((b-c) t^{\alpha}\right)^{-\alpha}\right)-1\right]}{-2 /(K(b-c))}+C_{5}}{K}\right. \\
& \left.-a\left(\frac{\left[\left(t^{\alpha} E_{\alpha, \alpha+1}\left((b-c) t^{\alpha}\right)^{-\alpha}\right)-1\right]}{-2 /(K(b-c))}+C_{5}\right)\right)+C_{6} .
\end{aligned}
$$

\subsection{Numerical simulations}

In this subsection, we put $\mathrm{a}=0.1, \mathrm{~b}=1, \mathrm{c}=0.5$ and $\mathrm{K}=20$ and consider numerical solutions in both ordinary-order and fractional-order Hanta-virus models [2]. The solutions $M_{i}$ and $M_{s}$ can be easily found if we consider solutions (4.3) and (4.4) of ordinary differential equation systems with the values above. Putting $M_{s}(0)=25$ and $M_{i}(0)=15$ conditions, we can find the constants $C_{1}$ and $C_{2}$ as

$$
\mathrm{C}_{1}=0.5753641449, \quad \mathrm{C}_{2}=-0.1777777778 .
$$

In this work all numerical calculations and simulations were carried out in Maple 18. We provide some graphical illustrations of the features of solutions for models with derivatives of both ordinary and fractional order. With the help of Hanta-virus solution as seen in Figures 1 and 3,

i) For $b>c$ and $K \leqslant \frac{b}{a(b-c)}$, we can see $(K(b-c), 0)$ equilibrium point is asymptotically determined

ii) For $b>c$ and $k>\frac{b}{a(b-c)}$, we can see

$$
\left(\frac{b}{a}, k(b-c)-\frac{b}{a}\right),
$$

this equilibrium point is asymptotically determined by a similar procedure $[2,3,10,18]$.

In Figure 2, with the help of initial conditions, arbitrary constant in the solution of equation (1.3) is taken $\mathrm{C}_{1} \cong 0.5753641449$.

Figures 4 and 5 show solutions graphics of $M_{s}$ and $M_{i}$ and density plots for different $\alpha$ values with fraction order. 

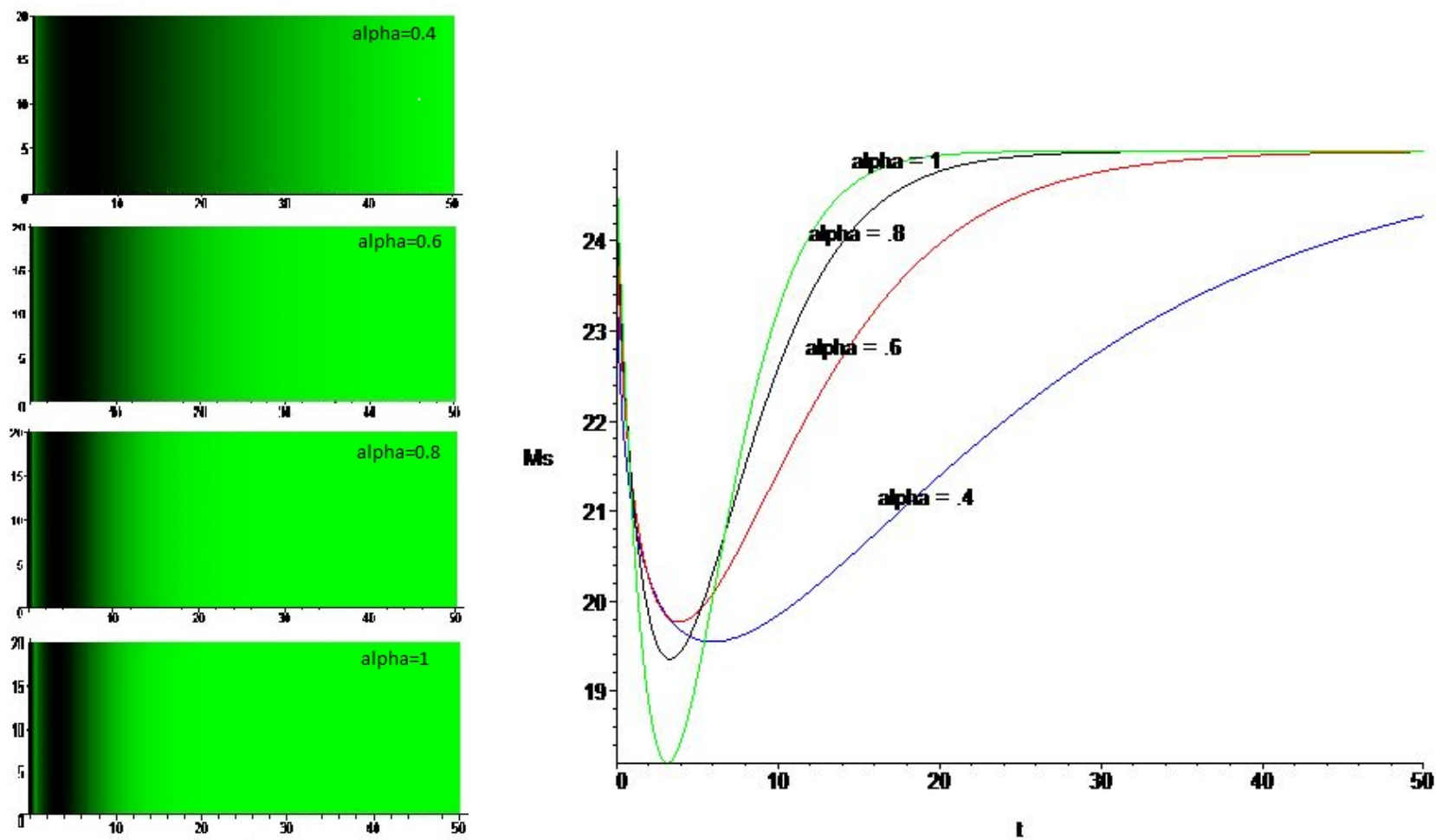

Figure 5: Density plots (left) and solutions of $M_{s}$ for different $\alpha$ values (right).

\section{Conclusions}

As one can clearly see from the article, Lie symmetry solutions of the ordinary differential equation can be easily found by using tangent vector. But for Lie symmetry solution of the differential equations or for Lie symmetry solutions of equation systems with fractional order, choices of arbitrary constants are hard and important, because they include Mittag-Leffler functions.

The arbitrary constants that are appropriate for given initial conditions guarantee positive solutions. However, for different initial conditions, because of the possibility of the negative solution, one should be cautious when choosing arbitrary constants.

\section{Acknowledgment}

The second author Mehmet KOCABIYIK would like to thank the Scientific and Technological Research Council of Turkey (TUBITAK 2228-A programme).

\section{References}

[1] F. A. Abdullah, A. I. M. Ismail, Simulations of the spread of the Hantavirus using fractional differential equations, Matematika (Johor Bahru), 27 (2011), 149-158. 1, 2.2

[2] G. Abramson, V. M. Kenkre, Spatiotemporal patterns in the hantavirus infection, Phys. Rev. E, 66 (2002), $011912.1,4.3$

[3] G. Abramson, V. M. Kenkre, T. L. Yates, R. R. Parmenter, Traveling waves of infection in the Hantavirus epidemics, Bull. Math. Biol., 65 (2003), 519-534. 1, 1, 4.3

[4] L. J. S. Allen, M. Langlais, C. J. Phillips, The dynamics of two viral infections in a single host population with applications to hantavirus, Math. Biosci., 186 (2003), 191-217. 1

[5] L. J. S. Allen, R. K. McCormack, C. B. Jonsson, Mathematical models for hantavirus infection in rodents, Bull. Math. Biol., 68 (2006), 511-524. 1

[6] D. Baleanu, H. Mohammadi, S. Rezapour, Positive solutions of an initial value problem for nonlinear fractional differential equations, Abstr. Appl. Anal., 2012 (2012), 7 pages. 3.1

[7] G. Bluman, Invariant solutions for ordinary differential equations, SIAM J. Appl. Math., 50 (1990), 1706-1715. 2 
[8] G. W. Bluman, S. Kumei, Symmetries and Differential Equations, Appl. Math. Sci., 81, Springer-Verlag, New York, (1989). 2

[9] M.-X. Chen, D. P. Clemence, Analysis of and numerical schemes for a mouse population model in hantavirus epidemics, J. Difference Equ. Appl., 12 (2006), 887-899. 1

[10] M.-X. Chen, D. P. Clemence, Stability properties of a nonstandard finite difference scheme for a hantavirus epidemic model, J. Difference Equ. Appl., 12 (2006), 1243-1256. 1, 4.3

[11] A. F. Cheviakov, GeM software package for computation of symmetries and conservation laws of differential equations, Comput. Phys. Comm., 176 (2007), 48-61. 2

[12] A. F. Cheviakov, Computation of fluxes of conservation laws, J. Engrg. Math., 66 (2010), 153-173.

[13] A. F. Cheviakov, Symbolic computation of local symmetries of nonlinear and linear partial and ordinary differential equations, Math. Comput. Sci., 4 (2010), 203-222.

[14] A. Cohen, An Introduction tO tHe Lie Theory of One-Parameter Groups; With Applications to the Solution of Differential Equations, D. C. Heath, Boston, New York, (1911). 2

[15] D.-Q. Ding, M. Qiang, X.-H. Ding, A non-standard finite difference scheme for an epidemic model with vaccination, J. Difference Equ. Appl., 19 (2013), 179-190. 1

[16] M. Edwards, M. C. Nucci, Application of Lie group analysis to a core group model for sexually transmitted diseases, J. Nonlinear Math. Phys., 13 (2006), 211-230. 2, 2.2

[17] R. K. Gazizov, A. A. Kasatkin, S. Y. Lukashchuk, Continuous transformation groups of fractional differential equations, Vestnik UGATU, 9 (2007), 125-135. 3.1, 3.1

[18] R. K. Gazizov, A. A. Kasatkin, S. Y. Lukashchuk, Group-invariant solutions of fractional differential equations; in: J. A. Tenreiro Machado (ed.) et al., Nonlinear Science and Complexity Springer, Berlin, (2011), 51-58. 1, 3.1, 3.1, 3.1, 3.1, 4.3

[19] S. M. Goh, A. I. M. Ismail, M. S. M. Noorani, I. Hashim, Dynamics of the hantavirus infection through variational iteration method, Nonlinear Anal. Real World Appl., 10 (2009), 2171-2176.

[20] A. Gökdoğan, M. Merdan, A. Yildirim, A multistage differential transformation method for approximate solution of Hantavirus infection model, Commun. Nonlinear Sci. Numer. Simul., 17 (2012), 1-8. 1, 2.2

[21] P. E. Hyden, Symmetry Methods for Differential Equations. A Beginner's Guide, Cambridge Texts Appl. Math, Cambridge University Press, Cambridge, (2000). 2, 3.1

[22] N. H. Ibragimov, Selected Works, Vol. 1, 2, Karlskrona, Sweden: Alga Publications, Blekinge Institute of Technology, (2001).

[23] N. H. Ibragimov, M. C. Nucci, Integration of third order ordinary differential equations by Lie's method: equations admitting three-dimensional Lie algebras, Lie Groups Appl., 1 (1994), 49-64 . 2, 2.2

[24] Z. G. Karadem, M. Yakıt Ongun, Logistic differential equations obtained from Hanta-virus model, (in Turkish) Fen Derg., 11 (2016), 82-91. 1

[25] A. A. Kilbas, H. M. Srivastava, J. J. Trujillo, Theory and Applications of Fractional Differential Equations, North-Holland Math. Stud., Elsevier, Amsterdam, (2006). 3.1, 3.1, 4.2

[26] B. K. Oldham, J. Spainer, The Fractional Calculus. Theory and Applications of Differentiation and Integration to Arbitrary Order, Math. Sci. Eng, 111, Academic Press , New York-London, (1974). 2.2, 3.1

[27] P. J. Oliver, Applications of Lie Groups to Differential Equations, Grad. Texts Math., 107, Springer-Verlag, New York, (1986). 2, 2.1

[28] L. V. Ovsiannikov, Group Analysis of Differential Equations, Academic Press, New York-London, (1982). 2, 3.1

[29] I. Podlubny, Fractional Differential Equations, An Introduction to Fractional Derivatives, Fractional Differential Equations, to Methods of Their Solution and Some of Their Applications, Math. Sci. Eng., Academic Press, San Diego, CA, (1999). $1,2.2,3.1$

[30] S. Z. Rida, A. A. El Radi, A. Arafa, M. Khalil, The effect of the environmental parameter on the Hantavirus infection through a fractional-order SI model, Int. J. Basic Appl. Sci., 1 (2012), 88-99. 1

[31] S. G. Samko, A. A. Kilbas, O. I. Marichev, Fractional Integrals And Derivatives. Theory and Applications, Gordon and Breach, Yverdon, (1993). 3.1

[32] V. Torrisi, M. C. Nucci, Application of Lie group analysis to a mathematical model which describes HIV transmission; in: J. A. Leslie and T. P. Robart (eds.), The Geometrical Study of Differential Equations, Washington, DC, (2000), Contemp. Math., 285, Amer. Math. Soc., Providence, RI, (2001), 11-20. 2

[33] C. L. Wesley, Discrete-time and continuous-time models with applications to the spread of hantavirus in wild rodent and human populations, Diss., Texas Tech, University, (2008). 1, 2.2

[34] S. Yüzbaş1, M. Sezer, An exponential matrix method for numerical solutions of Hantavirus infection model, Appl. Appl. Math., 8 (2013), 99-115. 1 